\title{
Best proximity points for semi-cyclic contraction pairs in regular cone metric spaces
}

\author{
M. Ahmadi Baseri* and H. Mazaheri \\ (Communicated by Nihal YILMAZ ÖZGÜR)
}

\begin{abstract}
The aim of this paper is to establish some conditions which guarantee the existence of best proximity for semi-cyclic contraction pairs in regular cone metric spaces. We obtain best proximity points and prove convergence results for such maps in regular cone metric spaces.
\end{abstract}

Keywords: Best proximity point; Regular cone metric; Semi-cyclic contraction pairs; Lower bound.

AMS Subject Classification (2010): Primary: 41A65; Secondary: 41A52; 46N10.

${ }^{*}$ Corresponding author

\section{Introduction and preliminaries}

Let $X:=(X, d)$ be a metric space and $A$ and $B$ be non-empty subsets of $X, \varphi:[0, \infty) \rightarrow[0, \infty)$ be a strictly increasing map and $S, T$ be two self mappings on $A \cup B$. The pair $(S, T)$ is called a semi-cyclic $\varphi$-contraction pair if $S(A) \subseteq B, T(B) \subseteq A$ and

$$
d(S x, T y) \leq d(x, y)-\varphi(d(x, y))+\varphi(d(A, B)),
$$

for all $x \in A$ and $y \in B$ [12]. When $S=T, T$ is called a $\varphi$-contraction map [1]. A semi-cyclic contraction pair is a semi-cyclic $\varphi$-contraction pair with $\varphi(t)=(1-k) t, k \in[0,1)$. In this case the pair $(S, T)$ satisfies for some $k \in(0,1)$,

$$
d(S x, T y) \leq k d(x, y)+(1-k) d(A, B)
$$

for all $x \in A$ and $y \in B$ [3]. When $S=T, T$ is called a cyclic contraction map. In 2006, Eldered and Veeramani obtained best proximity point results for cyclic contraction maps [2]. They raised a question and in 2009, Al-Thagafi and Shahzad answered it for cyclic $\varphi$-contraction maps [1]. Also, in 2012, Karapinar proved some theorems for generalized cyclic contraction maps [7].

In 2011, Gabeleh and Abkar proved a theorem on the existence and convergence of best proximity points for a semi-cyclic contraction pair $(S, T)$ [3]. Thakur and Sharma [12], obtained best proximity point results for semi-cyclic $\varphi$-contraction pair in 2014.

On the other hand, Huang and Zhang [6] introduced cone metric spaces as a generalization of metric spaces. In cone metric spaces the distance between two members not necessary a real positive, it can be sequence, function, matrix and any arbitrary Banach space. Hence achieved results is important and has many applications in sciences. In 2007, Rezapour [10] prove best proximity results in cone metric spaces. In 2011, Haghi et al [4] obtained best proximity points for cyclic contraction maps. In 2014, Lee [9] prove cone metric version of existence and convergence for best proximity points. Also, In 2015, Kumar and Som [8] give best proximity theorems in regular cone metric spaces. In this paper, we establish some conditions which guarantee the existence of best proximity for semi-cyclic contraction pairs in regular cone metric spaces. Then, we prove existence and convergence results for semi-cyclic contraction pair $(S, T)$ in regular cone metric spaces.

Received : 03-January-2017, Accepted : 21-June-2017 
To prove our results in the next section we recall some definitions and facts.

Definition 1.1. [6] Let $E$ be a real Banach space. A subset $P$ of $E$ is called a cone if and only if

(P1) $\mathrm{P}$ is closed, non-empty and $P \neq\{0\}$;

(P2) $a, b \in \mathbb{R}, a, b \geq 0$ and $x, y \in P$ implies $a x+b y \in P$;

(P3) $x \in P$ and $-x \in P$ implies $x=0$.

We define a partial ordering $\preceq$ with respect to $P$ by $x \preceq y$ if and only if $y-x \in P$. $x \prec y$ will stand for $x \preceq y$ and $x \neq y$, while $x \ll y$ will stand for $y-x \in \operatorname{int} P$, where int $P$ denotes the interior of $P$.

Definition 1.2. [6] Let $X$ be a non-empty set and $E$ be a Banach space. Suppose that a mapping $d: X \times X \rightarrow E$ satisfies:

(d1) $0 \preceq d(x, y)$ for every $x, y \in X$ and $d(x, y)=0$ if and only if $x=y$;

(d2) $d(x, y)=d(y, x)$ for every $x, y \in X$;

(d3) $d(x, y) \preceq d(x, z)+d(z, y)$ for every $x, y, z \in X$.

Then $d$ is called a cone metric and $(X, d)$ is called a cone metric space.

A map $f: P \rightarrow P$ is said to be increasing (strictly increasing) whenever $x \preceq y$ implies that $f(x) \preceq f(y)(x \prec y$ implies that $f(x) \prec f(y))$.

A continuous function $f: P \rightarrow P$ has a maximum point at $a$ if $f(x) \preceq f(a)$ for all $x \in P$. Similarly, the function has a minimum point at $a$ if $f(a) \preceq f(x)$ for all $x \in P$. The value of the function at a maximum point is called the maximum value of the function and the value of the function at a minimum point is called the minimum value of the function.

A cone $P$ is said to be normal if there is a number $M>0$ such that for all $x, y \in E$

$$
0 \preceq x \preceq y \quad \text { implies } \quad\|x\| \leq M\|y\| .
$$

The least positive number $M$ satisfying the above inequality is called the normal constant of $P$.

The cone $P$ is called regular if every increasing sequence which is bounded from above is convergent. That is, if $\left\{x_{n}\right\}_{n \geq 1}$ is a sequence such that $x_{1} \preceq x_{2} \preceq \ldots \preceq y$ for some $y \in E$, then there is $x \in E$ such that $\lim _{n \rightarrow \infty}\left\|x_{n}-x\right\|=0$. Equivalently the cone $P$ is regular if and only if every decreasing sequence which is bounded from below is convergent. Every regular cone is normal [11].

The following example shows that the category of regular cone metric spaces is bigger that the category of metric spaces.

Example 1.1. [5] Let $E=\left(L^{1}[0,1],\|\cdot\|_{1}\right), P=\{f \in E: f \succeq 0 \quad$ a.e. $\},(X, \rho)$ be a metric space and $d: X \times X \rightarrow E$ be defined by $d(x, y)=f_{x, y}$, where $f_{x, y}(t)=\rho(x, y) t^{2}$. Then $(X, d)$ is a regular cone metric space. In fact, if $\left\{f_{n}\right\}_{n \geq 1}$ is an increasing sequence and there is $g \in L^{1}$ such that $f_{1} \preceq f_{2} \preceq \ldots \preceq f_{n} \preceq \ldots \preceq g$ for all almost $x$, then $\left\{f_{n}\right\}_{n \geq 1}$ converges to a function $f$ for all almost $x$. Then, $f_{n} \preceq f \preceq g$ (a.e.) for all $n \geq 1$. Thus $g-f_{1} \in L^{1}, g-f_{n} \preceq g-f_{1}$ for all $n \geq 1$ and $\lim _{n \rightarrow \infty} g-f_{n}=g-f$ (a.e.). Hence by the Lebesgue dominated convergence theorem, $f \in L^{1}$ and $\lim _{n \rightarrow \infty}\left\|f_{n}-f\right\|_{1}=0$. So, the cone $P$ is regular.

Let $(X, d)$ be a cone metric space and $A$ be a non-empty subset of $X$. We say that $A$ is bounded whenever there is $e \gg 0$ such that $d(x, y) \preceq e$ for all $x, y \in A$.

Definition 1.3. [4] Let $A$ and $B$ be non-empty subsets of cone metric space $(X, d)$. An element $p \in P$ is said to be a lower bound for $A \times B$ whenever

$$
p \preceq d(a, b),
$$

for all $(a, b) \in A \times B$. If $p \succeq q$ for all lower bound $q$ for $A \times B$, then $p$ is called the greatest lower bound for $A \times B$. We denote it by $d(A, B)$.

Clearly, $d(A, B)$ is a unique vector in $P$.

Let $\left\{x_{n}\right\}$ be a sequence in a cone metric space $(X, d)$ and $x \in X$. If for every $c \in \operatorname{int} P$, there is a natural number $N$ such that for every $n>N, c-d\left(x_{n}, x\right) \in \operatorname{int} P$, then $\left\{x_{n}\right\}$ converges to $x$ with respect to $P$ and is denoted by $\lim _{n \rightarrow \infty} x_{n}=x$.

Lemma 1.1. [6] Let $(X, d)$ be a cone metric space, $P$ be a normal cone, $\left\{x_{n}\right\}$ and $\left\{y_{n}\right\}$ be sequences in $X$. Then 
(i) $x_{n}$ converges to $x$ with respect to $P$ if and only if $d\left(x_{n}, x\right) \rightarrow 0$ as $n \rightarrow \infty$,

(ii) If $x_{n} \rightarrow x$ and $y_{n} \rightarrow y$ as $n \rightarrow \infty$ with respect to $P$, then $d\left(x_{n}, y_{n}\right) \rightarrow d(x, y)$

as $n \rightarrow \infty$,

(iii) If $x_{n} \rightarrow x$ and $y_{n} \rightarrow y$ as $n \rightarrow \infty$ with respect to $P$ and $y_{n}-x_{n} \in P$ for

every $n \in \mathbb{N}$, then $y-x \in P$.

\section{Main results}

Throughout this section, $E$ is a normed space, $(X, d)$ is regular cone metric space, $\preceq$ is the partial ordering with respect of $P$ and $A, B$ are non-empty subsets of $X$.

Sequences Construction Consider $x_{0} \in A$, then $S x_{0} \in B$, so there exists $y_{0} \in B$ such that $y_{0}=S x_{0}$. Now $T y_{0} \in A$, so there exists $x_{1} \in A$ such that $x_{1}=T y_{0}$. Inductively, we define sequences $\left\{x_{n}\right\}$ and $\left\{y_{n}\right\}$ in $A$ and $B$, respectively by

$$
x_{n+1}=T y_{n}, \quad y_{n}=S x_{n} \text { for } n \in \mathbb{N} \cup\{0\} .
$$

Theorem 2.1. Let $S, T: A \cup B \rightarrow A \cup B$ be maps such that $S(A) \subseteq B, T(B) \subseteq A$ and

$$
d(S x, T y) \preceq(k / 3)\{d(x, y)+d(S x, x)+d(T y, y)\}+(1-k) d(a, b),
$$

for all $(a, b),(x, y) \in A \times B$, where $k \in(0,1)$ is a constant. Then $d(A, B)$ exists.

proof. Let $d_{n}=d\left(x_{n}, S x_{n}\right)$. By inequality (2.2),

$$
d_{n+1} \preceq(k / 3)\left\{d\left(y_{n}, x_{n+1}\right)+d_{n+1}+d\left(y_{n}, x_{n+1}\right)\right\}+(1-k) d(a, b) .
$$

Since

$$
d\left(y_{n}, x_{n+1}\right) \preceq(k / 3)\left\{2 d_{n}+d\left(y_{n}, x_{n+1}\right)\right\}+(1-k) d(a, b),
$$

hence

$$
d\left(y_{n}, x_{n+1}\right) \preceq \frac{(2 k / 3)}{(1-(k / 3))} d_{n}+\frac{(1-k)}{(1-(k / 3))} d(a, b) .
$$

Therefore

$$
\begin{aligned}
d_{n+1} & \preceq(2 k / 3) \frac{(2 k / 3)}{(1-(k / 3))} d_{n}+(2 k / 3) \frac{(1-k)}{(1-(k / 3))} d(a, b) \\
& +(k / 3) d_{n+1}+(1-k) d(a, b) .
\end{aligned}
$$

Then

$$
d_{n+1} \preceq \frac{\left(4 k^{2} / 9\right)}{(1-(k / 3))^{2}} d_{n}+\frac{(1-k)(1+k / 3)}{(1-(k / 3))^{2}} d(a, b),
$$

which implies that

$$
d_{n+1} \preceq \alpha d_{n}+(1-\alpha) d(a, b),
$$

for all $(a, b) \in A \times B$, where $\alpha=\left(4 k^{2} / 9\right) /\left((1-(k / 3))^{2}\right) \in(0,1)$. It follows that $d_{n+1} \preceq d_{n}$. By the regularity of $P$, there exists $p \in P$ such that $\lim _{n \rightarrow \infty} d_{n}=p$. Thus $p \preceq d(a, b)$ holds for any $(a, b)$ in $A \times B$. Now if $q$ is a lower bound for $A \times B$, then $q \preceq d_{n}$ for all $n \in \mathbb{N} \cup\{0\}$. So $q \preceq p$. Therefore, $d(A, B)=p$.

Note that, the inequality (2.2) is equivalent to

$$
d(S x, T y) \leq(k / 3)\{d(x, y)+d(S x, x)+d(T y, y)\}+(1-k) d(A, B)
$$

in metric spaces.

Theorem 2.2. Suppose that the conditions of Theorem 2.1 hold, for $x_{0} \in A$, the sequences $\left\{x_{n}\right\}$ and $\left\{y_{n}\right\}$ are generated by (2.1). If $\left\{x_{n}\right\}$ and $\left\{y_{n}\right\}$ respectively have a convergent subsequence in $A$ and $B$, then there exists $x \in A$ and $y \in B$ such that

$$
d(x, S x)=d(A, B)=d(y, T y) .
$$


proof. Set $d_{n}=d\left(x_{n}, S x_{n}\right)$. Let $\left\{y_{n_{k}}\right\}$ be a subsequence of $\left\{y_{n}\right\}$ such that $y_{n_{k}} \rightarrow y$. The relation

$$
p=d(A, B) \preceq d\left(T y_{n_{k}}, y\right) \preceq d\left(y_{n_{k}}, y\right)+d\left(y_{n_{k}}, T y_{n_{k}}\right)
$$

holds for each $k \geq 1$. Since

$$
p=d(A, B) \preceq d\left(y_{n_{k}}, T y_{n_{k}}\right) \preceq \alpha d_{n_{k}}+(1-\alpha) d(a, b),
$$

for all $(a, b) \in A \times B$, where $\alpha=(2 k / 3) /(1-(k / 3)) \in(0,1)$. It follows that $p=d(A, B) \preceq d\left(y_{n_{k}}, T y_{n_{k}}\right) \preceq d_{n_{k}}$. Since $\left\{d\left(S x_{n_{k}}, x_{n_{k}}\right)\right\}$ is a subsequence of $\left\{d_{n}\right\}$, hence $\lim _{k \rightarrow \infty} d\left(S x_{n_{k}}, x_{n_{k}}\right)=p$. Thus $\lim _{k \rightarrow \infty} d\left(y_{n_{k}}, T y_{n_{k}}\right)=p$. So $d\left(T y_{n_{k}}, y\right) \rightarrow p$ as $k \rightarrow \infty$. Now, for $k \geq 1$,

$$
\begin{aligned}
d\left(T y, y_{n_{k}}\right) & \preceq(k / 3)\left\{d\left(y, x_{n_{k}}\right)+d\left(S x_{n_{k}}, x_{n_{k}}\right)+d(T y, y)\right\}+(1-k) d(a, b) \\
& \preceq(k / 3)\left\{2 d\left(y, y_{n_{k}}\right)+2 d\left(y_{n_{k}}, x_{n_{k}}\right)+d\left(T y, y_{n_{k}}\right)\right\}+(1-k) d(a, b) .
\end{aligned}
$$

Thus

$$
p=d(A, B) \preceq d\left(T y, y_{n_{k}}\right) \preceq \alpha\left\{d\left(y, y_{n_{k}}\right)+d\left(y_{n_{k}}, x_{n_{k}}\right)\right\}+(1-k) d(a, b),
$$

for all $(a, b) \in A \times B$, where $\alpha=((2 k) / 3) /(1-(k / 3)) \in(0,1)$. Therefore, by relation (2.3), $d(T y, y)=p=d(A, B)$. Similarly, it can be proved that $d(x, S x)=d(A, B)$.

Example 2.1. Let $E=\mathbb{R}^{2}, P=\{(x, y) \in E: \quad x, y \geq 0\} \subset \mathbb{R}^{2}, X=\mathbb{R}$ and $d: X \times X \rightarrow E$ be such that $d(x, y)=(|x-y|, \lambda|x-y|)$, where $\lambda \geq 0$ is a constant. Let $A=[0,1], B=[-1,0]$. So $d(A, B)=0$. Define $S, T: A \cup B \rightarrow A \cup B$ by

$$
S(x)=\left\{\begin{array}{ll}
\frac{-x}{2}, & x \in A \\
\frac{x}{2}, & x \in B,
\end{array} \quad T(x)= \begin{cases}\frac{x}{2}, & x \in A \\
\frac{-x}{2}, & x \in B .\end{cases}\right.
$$

then for all $(a, b),(x, y) \in A \times B$ and $k=7 / 10$,

$$
\begin{aligned}
(7 / 30) & \{d(x, y)+d(S x, x)+d(T y, y)\}+(3 / 10) d(a, b)-d(S x, T y) \\
& =(7 / 30)\{(|x-y|, \lambda|x-y|)+(3|x| / 2,3 \lambda|x| / 2)+(3|y| / 2,3 \lambda|y| / 2)\} \\
& +(3 / 10)(|a-b|, \lambda|a-b|)-(1 / 2)(|x-y|, \lambda|x-y|) \\
& =((-8 / 30)|x-y|+3|x| / 2+3|y| / 2+(3 / 10)|a-b|, \lambda((-8 / 30)|x-y|+3|x| / 2 \\
& +3|y| / 2+(3 / 10)|a-b|)) \in P .
\end{aligned}
$$

Hence for all $(a, b),(x, y) \in A \times B$,

$$
d(S x, T y) \preceq(7 / 30)\{d(x, y)+d(S x, x)+d(T y, y)\}+(3 / 10) d(a, b) .
$$

So $d(A, B)=0$. Therefore $x=0$ and $y=0$ are best proximity points for $S$ and $T$ respectively.

Theorem 2.3. Let $S, T: A \cup B \rightarrow A \cup B$ be maps such that $S(A) \subseteq B, T(B) \subseteq A$ and

$$
d(S x, T y) \preceq k \max \{d(x, y),(1 / 2)\{d(S x, x)+d(T y, y)\}\}+(1-k) d(a, b),
$$

for all $(a, b),(x, y) \in A \times B$, where $k \in(0,1)$ is a constant. Then $d(A, B)$ exists.

proof. Assume that $\max \{d(x, y),(1 / 2)\{d(S x, x)+d(T y, y)\}\}=d(x, y)$. So $(1 / 2)\{d(S x, x)+d(T y, y)\} \preceq d(x, y)$. Set $d_{n}=d\left(x_{n}, S x_{n}\right)$. Since

$$
\begin{aligned}
d_{n+1} & \preceq k d\left(y_{n}, x_{n+1}\right)+(1-k) d(a, b) \\
& \preceq k^{2} d_{n}+\left(1-k^{2}\right) d(a, b),
\end{aligned}
$$

for all $(a, b)$ in $A \times B$. It follows that $d_{n+1} \preceq d_{n}$.

Assume that $\max \{d(x, y),(1 / 2)\{d(S x, x)+d(T y, y)\}\}=(1 / 2)\{d(S x, x)+d(T y, y)\}$. So $d(x, y) \preceq(1 / 2)\{d(S x, x)+$ $d(T y, y)\}$. Thus

$$
d_{n+1} \preceq(k / 2)\left\{d_{n+1}+d\left(y_{n}, x_{n+1}\right\}+(1-k) d(a, b) .\right.
$$


Since

$$
d\left(y_{n}, x_{n+1}\right) \preceq(k / 2)\left\{d_{n}+d\left(y_{n}, x_{n+1}\right)\right\}+(1-k) d(a, b),
$$

hence

Therefore

$$
d\left(y_{n}, x_{n+1}\right) \preceq \frac{(k / 2)}{1-(k / 2)} d_{n}+\frac{(1-k)}{1-(k / 2)} d(a, b) .
$$

which implies that

$$
d_{n+1} \preceq \frac{\left(k^{2} / 4\right)}{(1-(k / 2))^{2}} d_{n}+\frac{(1-k)}{(1-(k / 2))^{2}} d(a, b),
$$

$$
d_{n+1} \preceq \alpha d_{n}+(1-\alpha) d(a, b),
$$

for all $(a, b),(x, y) \in A \times B$, where $\alpha=\left(k^{2} / 4\right) /\left((1-(k / 2))^{2}\right) \in(0,1)$. It follows that $d_{n+1} \preceq d_{n}$. Next, the proof continues similar to the proof of Theorem 2.1.

Note that, the inequality (2.4) is equivalent to

$$
d(S x, T y) \leq k \max \{d(x, y),(1 / 2)\{d(S x, x)+d(T y, y)\}\}+(1-k) d(A, B)
$$

in metric spaces.

Theorem 2.4. Suppose that the conditions of Theorem 2.3 hold, for $x_{0} \in A$, the sequences $\left\{x_{n}\right\}$ and $\left\{y_{n}\right\}$ are generated by (2.1). If $\left\{x_{n}\right\}$ and $\left\{y_{n}\right\}$ respectively have a convergent subsequence in $A$ and $B$, then there exists $x \in A$ and $y \in B$ such that

$$
d(x, S x)=d(A, B)=d(y, T y) .
$$

proof. Set $d_{n}=d\left(x_{n}, S x_{n}\right)$. Let $\left\{y_{n_{k}}\right\}$ be a subsequence of $\left\{y_{n}\right\}$ such that $y_{n_{k}} \rightarrow y$. The relation

$$
p=d(A, B) \preceq d\left(T y_{n_{k}}, y\right) \preceq d\left(y_{n_{k}}, y\right)+d\left(y_{n_{k}}, T y_{n_{k}}\right)
$$

holds for each $k \geq 1$.

Assume that $\max \{d(x, y),(1 / 2)\{d(S x, x)+d(T y, y)\}\}=d(x, y)$. So $(1 / 2)\{d(S x, x)+d(T y, y)\} \preceq d(x, y)$. Thus

$$
d\left(y_{n_{k}}, T y_{n_{k}}\right) \preceq k d_{n_{k}}+(1-k) d(a, b),
$$

for all $(a, b) \in A \times B$. It follows that $d\left(y_{n_{k}}, T y_{n_{k}}\right) \preceq d_{n_{k}}$. Since $\left\{d\left(S x_{n_{k}}, x_{n_{k}}\right)\right\}$ is a subsequence of $\left\{d_{n}\right\}$, hence $\lim _{k \rightarrow \infty} d\left(S x_{n_{k}}, x_{n_{k}}\right)=p$. Thus

$$
\lim _{k \rightarrow \infty} d\left(y_{n_{k}}, T y_{n_{k}}\right)=p
$$

So $d\left(T y_{n_{k}}, y\right) \rightarrow p$ as $k \rightarrow \infty$. Now, for each $k \geq 1$

$$
\begin{aligned}
d\left(T y, y_{n_{k}}\right) & \preceq k d\left(y, x_{n_{k}}\right)+(1-k) d(a, b) \\
& \preceq k\left\{d\left(y, y_{n_{k}}\right)+d\left(y_{n_{k}}, x_{n_{k}}\right)\right\}+(1-k) d(a, b) .
\end{aligned}
$$

i.e.

$$
p=d(A, B) \preceq d\left(T y, y_{n_{k}}\right) \preceq k\left\{d\left(y, y_{n_{k}}\right)+d_{n_{k}}\right\}+(1-k) d(a, b),
$$

for all $(a, b) \in A \times B$. Letting $k \rightarrow \infty$, we have $d(T y, y)=p=d(A, B)$.

Assume that $\max \{d(x, y),(1 / 2)\{d(S x, x)+d(T y, y)\}\}=(1 / 2)\{d(S x, x)+d(T y, y)\}$. So $(1 / 2)\{d(S x, x)+d(T y, y)\} \preceq$ $d(x, y)$. Thus

which implies that

$$
d\left(y_{n_{k}}, T y_{n_{k}}\right) \preceq(k / 2)\left\{d_{n_{k}}+d\left(y_{n_{k}}, T y_{n_{k}}\right)\right\}+(1-k) d(a, b),
$$

$$
d\left(y_{n_{k}}, T y_{n_{k}}\right) \preceq \alpha d_{n_{k}}+(1-\alpha) d(a, b),
$$

for all $(a, b) \in A \times B$, where $\alpha=(k / 2) /(1-(k / 2)) \in(0,1)$. It follows that, $d\left(y_{n_{k}}, T y_{n_{k}}\right) \preceq d_{n_{k}}$. Since $\lim _{k \rightarrow \infty} d_{n_{k}}=p$, hence $d\left(y_{n_{k}}, T y_{n_{k}}\right) \rightarrow p$ as $k \rightarrow \infty$. So $\lim _{k \rightarrow \infty} d\left(T y_{n_{k}}, y\right)=p$. Now, for each $k \geq 1$

$$
\begin{aligned}
d\left(T y, y_{n_{k}}\right) & \preceq(k / 2)\left\{d\left(y_{n_{k}}, x_{n_{k}}\right)+(T y, y)\right\}+(1-k) d(a, b) \\
& \preceq(k / 2)\left\{d_{n_{k}}+d\left(T y, y_{n_{k}}\right)+d\left(y_{n_{k}}, y\right)\right\}+(1-k) d(a, b) .
\end{aligned}
$$

So

$$
\left(T y, y_{n_{k}}\right) \preceq \alpha\left\{d_{n_{k}}+d\left(y_{n_{k}}, y\right)\right\}+(1-\alpha) d(a, b),
$$

for all $(a, b) \in A \times B$, where $\alpha=(k / 2) /(1-(k / 2)) \in(0,1)$. Letting $k \rightarrow \infty$, we have $d(T y, y)=p=d(A, B)$. Similarly, it can be proved that $d(x, S x)=d(A, B)$. 
Example 2.2. Suppose that the conditions of Example 2.1 hold. So for all $(a, b),(x, y) \in A \times B$ and $k=6 / 10$, $\max \{d(x, y),(1 / 2)\{d(S x, x)+d(T y, y)\}\}=d(x, y)$. Thus

$$
\begin{aligned}
& (6 / 10) d(x, y)+(4 / 10) d(a, b)-d(S x, T y) \\
& \quad=((1 / 10)|x-y|+(4 / 10)|a-b|, \lambda((1 / 10)|x-y|+(4 / 10)|a-b|)) \in P .
\end{aligned}
$$

Hence for all $(a, b),(x, y) \in A \times B$,

$$
d(S x, T y) \preceq(6 / 10) \max \{d(x, y),(1 / 2)\{d(S x, x)+d(T y, y)\}\}+(4 / 10) d(a, b) .
$$

So $d(A, B)=0$. Therefore $x=0$ and $y=0$ are best proximity points for $S$ and $T$ respectively.

Theorem 2.5. Let $\varphi: P \rightarrow P$ be a strictly increasing map, $S, T: A \cup B \rightarrow A \cup B$ be maps satisfying $S(A) \subseteq B, T(B) \subseteq A$ and

$$
d(S x, T y) \preceq d(x, y)-\varphi(d(x, y))+\varphi(p),
$$

for all $(x, y) \in A \times B$, where $p$ is a lower bound for $A \times B$. Then, $d(A, B)=p$.

proof. Let $d_{n}=d\left(x_{n}, S x_{n}\right)$. Then, $d_{n+1} \preceq d_{n}$. By the regularity of $P$, there exists $q \in P$ such that $\lim _{n \rightarrow \infty} d_{n}=q$. Since $\varphi$ be a strictly increasing map and $p$ is a lower bound for $A \times B$. Hence $\varphi(p) \preceq \varphi\left(d\left(y_{n}, x_{n+1}\right)\right)$. So

$$
\varphi\left(d\left(y_{n}, x_{n+1}\right)\right)-\varphi(p) \in P .
$$

By inequality (2.5),

$$
d\left(y_{n}, x_{n+1}\right)-\varphi\left(d\left(y_{n}, x_{n+1}\right)\right)+\varphi(p)-d_{n+1} \in P .
$$

From (2.5) and (2.6),

$$
d\left(y_{n}, x_{n+1}\right)-d_{n+1} \in P .
$$

So $d_{n+1} \preceq d\left(y_{n}, x_{n+1}\right)$. Since

$$
\begin{aligned}
d_{n+1} & \preceq d\left(y_{n}, x_{n+1}\right) \\
& \preceq d_{n}-\varphi\left(d_{n}\right)+\varphi(p)
\end{aligned}
$$

Letting $n \rightarrow \infty$, we have $\lim _{n \rightarrow \infty} \varphi\left(d_{n}\right)=\varphi(p)$. Since $p \preceq d_{n}$. Hence, $\varphi(p) \preceq \varphi(q) \preceq \varphi\left(d_{n}\right)$. Therefore $\varphi(p)=\varphi(q)$. It implies that $p=q$ and so $d(A, B)=p$.

Theorem 2.6. Suppose that the conditions of Theorem 2.5 hold, for $x_{0} \in A$, the sequences $\left\{x_{n}\right\}$ and $\left\{y_{n}\right\}$ are generated by (2.1). If $\left\{x_{n}\right\}$ and $\left\{y_{n}\right\}$ respectively have a convergent subsequence in $A$ and $B$, then there exists $x \in A$ and $y \in B$ such that

$$
d(x, S x)=d(A, B)=d(y, T y) .
$$

proof. Set $d_{n}=d\left(x_{n}, S x_{n}\right)$. Let $\left\{y_{n_{k}}\right\}$ be a subsequence of $\left\{y_{n}\right\}$ such that $y_{n_{k}} \rightarrow y$. The relation

$$
p=d(A, B) \preceq d\left(T y_{n_{k}}, y\right) \preceq d\left(y_{n_{k}}, y\right)+d\left(y_{n_{k}}, T y_{n_{k}}\right)
$$

holds for each $k \geq 1$. Since

$$
d\left(y_{n_{k}}, T y_{n_{k}}\right) \preceq d_{n_{k}} .
$$

Hence $\lim _{k \rightarrow \infty} d\left(y_{n_{k}}, T y_{n_{k}}\right)=p$. Thus $d\left(T y_{n_{k}}, y\right) \rightarrow p$ as $k \rightarrow \infty$. Now, for each $k \geq 1$

$$
\begin{aligned}
d\left(T y, y_{n_{k}}\right) & \preceq d\left(y, x_{n_{k}}\right) \\
& \preceq d\left(y, y_{n_{k}}\right)+d\left(y_{n_{k}}, x_{n_{k}}\right) .
\end{aligned}
$$

Letting $k \rightarrow \infty$, we have $d(T y, y)=p=d(A, B)$.

Similarly, it can be proved that $d(x, S x)=d(A, B)$. 
Example 2.3. Suppose that the conditions of Example 2.1 hold. Define $\varphi\left(t_{1}, t_{2}\right)=\left(t_{1}{ }^{2} /\left(1+2 t_{1}\right), t_{2}{ }^{2} /\left(1+2 t_{2}\right)\right)$ for $t_{1}, t_{2} \geq 0$. Because $p$ is a lower bound for $A \times B$. Then, $p=(0,0)$. Put $t=|x-y|$. So for all $(a, b),(x, y) \in A \times B$,

$$
\begin{aligned}
d(x, y) & -\varphi(d(x, y))+\varphi(p)-d(S x, T y) \\
& =(t, \lambda t)+\left(t^{2} /(1+2 t), \lambda^{2} t^{2} /(1+2 \lambda t)-(1 / 2)(t, \lambda t) \in P .\right.
\end{aligned}
$$

Hence for all $(x, y) \in A \times B$,

$$
d(S x, T y) \preceq d(x, y)-\varphi(d(x, y))+\varphi(p) .
$$

So $d(A, B)=0$. Therefore $x=0$ and $y=0$ are best proximity points for $S$ and $T$ respectively.

Theorem 2.7. Let $\varphi: P \rightarrow P$ be a strictly increasing map, $S, T: A \cup B \rightarrow A \cup B$ be maps satisfying $S(A) \subseteq B, T(B) \subseteq A$ and

$$
\begin{aligned}
d(S x, T y) & \preceq(1 / 3)\{d(x, y)+d(S x, x)+d(T y, y)\} \\
& -\varphi(d(x, y)+d(S x, x)+d(T y, y))+\varphi(p),
\end{aligned}
$$

for all $(x, y) \in A \times B$, where $p$ is a lower bound for $A \times B$. Then, $d(A, B)=p$.

proof. For a strictly increasing mapping $\varphi: P \rightarrow P$

$$
\begin{aligned}
\varphi(p) & \preceq \varphi(d(x, y)) \\
& \preceq \varphi(d(x, y)+d(S x, x)+d(T y, y)),
\end{aligned}
$$

for all $(x, y) \in A \times B$, so that

$$
d(S x, T y) \preceq(1 / 3)\{d(x, y)+d(S x, x)+d(T y, y)\} .
$$

Thus we have

$$
\begin{aligned}
d\left(x_{n}, S x_{n}\right) & \preceq(1 / 3)\left\{d\left(x_{n}, y_{n-1}\right)+d\left(S x_{n}, x_{n}\right)+d\left(T y_{n-1}, y_{n-1}\right)\right\} \\
& =(2 / 3) d\left(x_{n}, y_{n-1}\right)+(1 / 3) d\left(S x_{n}, x_{n}\right) .
\end{aligned}
$$

Since

$$
\begin{aligned}
d\left(x_{n}, y_{n-1}\right) & \preceq(1 / 3)\left\{d\left(y_{n-1}, x_{n-1}\right)+d\left(y_{n-1}, x_{n}\right)+d\left(y_{n-1}, x_{n-1}\right)\right\} \\
& =(2 / 3) d\left(y_{n-1}, x_{n-1}\right)+(1 / 3) d\left(y_{n-1}, x_{n}\right),
\end{aligned}
$$

hence

$$
\begin{aligned}
d\left(x_{n}, y_{n-1}\right) & \preceq d\left(y_{n-1}, x_{n-1}\right) \\
& =d\left(x_{n-1}, S x_{n-1}\right) .
\end{aligned}
$$

So

$$
d\left(x_{n}, S x_{n}\right) \preceq(2 / 3) d\left(x_{n-1}, S x_{n-1}\right)+(1 / 3) d\left(S x_{n}, x_{n}\right) .
$$

Therefore

$$
d\left(x_{n}, S x_{n}\right) \preceq d\left(x_{n-1}, S x_{n-1}\right) .
$$

Let $d_{n}=d\left(x_{n}, S x_{n}\right)$. Then $d_{n+1} \preceq d_{n}$ for $n \in \mathbb{N} \cup\{0\}$. By the regularity of $P$, there exists $q \in P$ such that $\lim _{n \rightarrow \infty} d_{n}=q$. Since

$$
\begin{aligned}
d_{n+1} & \preceq(1 / 3)\left\{d\left(x_{n+1}, y_{n}\right)+d_{n+1}\right\}-\varphi\left(2 d_{n}+d\left(x_{n+1}, y_{n}\right)\right)+\varphi(p) \\
& \preceq(2 / 3) d_{n}+(1 / 3) d_{n+1}-\varphi\left(d_{n}\right)+\varphi(p) .
\end{aligned}
$$

Hence

$$
\varphi\left(d_{n}\right)-\varphi(p) \preceq(2 / 3)\left\{d_{n}-d_{n+1}\right\} .
$$

Therefore $\lim _{n \rightarrow \infty} \varphi\left(d_{n}\right)=\varphi(p)$. Since $p \preceq d_{n}$. Hence, $p \preceq q$ and $\varphi(p) \preceq \varphi(q) \preceq \varphi\left(d_{n}\right)$. Thus, $\varphi(p)=\varphi(q)$. It implies that $p=q$ and so $d(A, B)=p$. 
Theorem 2.8. Suppose that the conditions of Theorem 2.7 hold, for $x_{0} \in A$, the sequences $\left\{x_{n}\right\}$ and $\left\{y_{n}\right\}$ are generated by (2.1). If $\left\{x_{n}\right\}$ and $\left\{y_{n}\right\}$ respectively have a convergent subsequence in $A$ and $B$, then there exists $x \in A$ and $y \in B$ such that

$$
d(x, S x)=d(A, B)=d(y, T y) .
$$

proof. Set $d_{n}=d\left(x_{n}, S x_{n}\right)$. Let $\left\{y_{n_{k}}\right\}$ be a subsequence of $\left\{y_{n}\right\}$ such that $y_{n_{k}} \rightarrow y$. The relation

$$
p=d(A, B) \preceq d\left(T y_{n_{k}}, y\right) \preceq d\left(y_{n_{k}}, y\right)+d\left(y_{n_{k}}, T y_{n_{k}}\right),
$$

holds for each $k \geq 1$. Since

$$
d\left(y_{n_{k}}, T y_{n_{k}}\right) \preceq(2 / 3) d_{n_{k}}+(1 / 3) d\left(y_{n_{k}}, T y_{n_{k}}\right) .
$$

Hence

$$
d\left(y_{n_{k}}, T y_{n_{k}}\right) \preceq d_{n_{k}} .
$$

It follows that $\lim _{k \rightarrow \infty} d\left(y_{n_{k}}, T y_{n_{k}}\right)=p$. Thus $d\left(T y_{n_{k}}, y\right) \rightarrow p$ as $k \rightarrow \infty$. Now, for $k \geq 1$

$$
\begin{aligned}
d\left(T y, y_{n_{k}}\right) & \preceq(1 / 3)\left\{d\left(y, x_{n_{k}}\right)+d\left(S x_{n_{k}}, x_{n_{k}}\right)+d(T y, y)\right\} \\
& \preceq(1 / 3)\left\{2 d\left(y, y_{n_{k}}\right)+2 d\left(y_{n_{k}}, x_{n_{k}}\right)+d\left(T y, y_{n_{k}}\right)\right\} .
\end{aligned}
$$

Thus

$$
d\left(T y, y_{n_{k}}\right) \preceq\left\{d\left(y, y_{n_{k}}\right)+d\left(y_{n_{k}}, x_{n_{k}}\right)\right\} .
$$

Therefore, $d(T y, y)=p=d(A, B)$.

Similarly, it can be proved that $d(x, S x)=d(A, B)$.

Example 2.4. Suppose that the conditions of Example 2.1 hold. Define $\varphi\left(t_{1}, t_{2}\right)=\left(t_{1}{ }^{2} /\left(1+8 t_{1}\right), t_{2}{ }^{2} /\left(1+8 t_{2}\right)\right)$ for $t_{1}, t_{2} \geq 0$. Because $p$ is a lower bound for $A \times B$. Then, $p=(0,0)$. So for all $(x, y) \in A \times B$,

$$
(1 / 3)\{d(x, y)+d(S x, x)+d(T y, y)\}-\varphi(d(x, y)+d(S x, x)+d(T y, y))+\varphi(p) \in P .
$$

Hence for all $(x, y) \in A \times B$,

$$
\begin{aligned}
d(S x, T y) & \preceq(1 / 3)\{d(x, y)+d(S x, x)+d(T y, y)\} \\
& -\varphi(d(x, y)+d(S x, x)+d(T y, y))+\varphi(p) .
\end{aligned}
$$

So $d(A, B)=0$. Therefore $x=0$ and $y=0$ are best proximity points for $S$ and $T$ respectively.

\section{References}

[1] Al-Thagafi, M. A., Shahzad, N., Convergence and existence result for best proximity points. Nonliner Analysis, Theory, Methods and Applications. 70 (2009), no. 10, 3665-3671.

[2] Eldred, A. A., Veeramani, P., Existence and convergence of best proximity points. J. Math. Anal. Appl. 323 (2006), no. 2, 1001-1006.

[3] Gabeleh, M., Abkar, A., Best proximity points for semi-cyclic contractive pairs in Banach spaces. Int. Math. Forum. 6 (2011), no. 44, 2179-2186.

[4] Haghi, R. H., Rakoc̃ević, V., Rezapour, Sh., Shahzad, N., Best proximity result in regular cone metric space. Rendiconti del circolo Mathematico di palermo Co. 60 (2011), no. 3, 323-327.

[5] Haghi, R. H., Rezapour, Sh., Fixed points of multifunctions on regular cone metric space. Expo. Math. 28 (2010), no. 1, 71-77.

[6] Huang, L. G., Zhang, X., Cone metric spaces and fixed point theorems of contractive mappings. J. Math. Anal. Appl. 332 (2007), no. 2, 1468-1476.

[7] Karapinar, E., Best proximity points of cyclic mappings. Appl. Math. 25 (2012), no. 11, 1761-1766.

[8] Kumar, L., Som T., Existence of best proximity points in regular cone Metric Spaces. Azerbaijan Journal of Mathematics. 5 (2015), no. 1, 44-53. 
[9] Lee, B. S., Cone metric version of existence and convergence for best proximity points. Universal J. Appl. Math. 2 (2014), no. 2, 104-108.

[10] Rezapour, Sh., Best approximations in cone metric spaces. Mathematica moravica. 11 (2007), 85-88.

[11] Rezapour, Sh., Hamlbarani Haghi, R., Some notes on the paper "Cone metric spaces and fixed point theorems of contractive mappings". J. Math. Anal. Appl. 345 (2008) no. 2, 719-724.

[12] Thakur, B. S., Sharma, A., Existence and convergence of best proximity points for semi-cyclic contraction pairs. International Journal of Analysis and Applications. 5 (2014), no. 1, 33-44.

\section{Affiliations}

M. AHMADI BASERI

AdDRESS: Department of Mathematics, Yazd University, Yazd, Iran.

E-MAIL: m.ahmadi@stu.yazd.ac.ir

ORCID ID: orcid.org/0000-0003-4997-6576

H. MAZAHERI

ADDRESS: Department of Mathematics, Yazd University, Yazd, Iran.

E-MAIL: hmazaheri@yazd.ac.ir

ORCID ID: orcid.org/0000-0003-3450-3776 